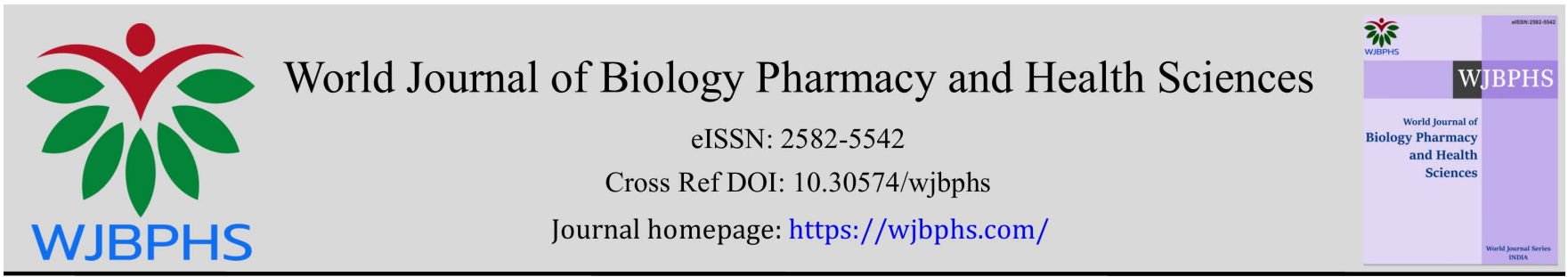

(CASE REPORT)

\title{
Mesenteric cystic lymphangioma - A rare case report; National Center for Pediatric Surgery, Gezira State, Sudan; 2021
}

Omer Taha Ahmed Elmukashfi 1,*, Taha Ahmed Elmukashfi Elsheikh 2, Ahmed Mustafa Idris Mohamed 1, Wala Osman Eltieb Omer ${ }^{1}$, Marwa Ahmed Ismail Elawal ${ }^{1}$, Samah Eltayeb Ahmed Nuwar ${ }^{1}$ and Diaaeldinn Yaseen Salman Mohammed ${ }^{3}$

${ }^{1}$ General Surgery Council, Sudan Medical Specialization Board, Khartoum, Sudan.

${ }^{2}$ Department of Community Medicine, Faculty of Medicine, University of Khartoum, Sudan.

${ }^{3}$ Department of Pediatric Surgery, Faculty of Medicine, University of Gezira, Sudan.

World Journal of Biology Pharmacy and Health Sciences, 2021, 08(02), 014-018

Publication history: Received on 07 September 2021; revised on 18 October 2021; accepted on 20 October 2021

Article DOI: https://doi.org/10.30574/wjbphs.2021.8.2.0095

\begin{abstract}
Cystic lymphangioma is a benign congenital malformation of the lymphatic vessels. At the National Center for Pediatric Surgery, Gezira State, Sudan; 2021; a female baby of 15 months old age presented complaining of abdominal distention of 10 days duration. Patient Looks unwell, not febrile, and not pale or jaundice; abdomen was distended with full flanks, there was a palpable mass in the epigastric region, no area of tenderness and no palpable organs. Abdomino-pelvic U/S showed very huge abdomino -pelvic cystic mass with multiple thick septa. CT abdomen showed huge abdomino-pelvic mass with multiple cysts that having variable sizes measuring up to $6 * 7 \mathrm{~cm}$. An elective explorative laparotomy was done. Intra operative findings: Multiple cystic masses arise from the root of the small bowel mesentery matted together with multiple enlarged mesenteric lymph nodes involving segment of distal jejunum with multiple adhesions. Release of adhesion, resection of the masses along with $57 \mathrm{~cm}$ of the involving jejunum, anastomosis was done, and homeostasis was secured. Histopathology result showed dilated cystic spaces; lined by flat endothelial cells and multiple reactive lymph nodes, no evidence of malignancy. Features are in line with lymphangioma and associated mesenteric lymphadenitis. Discussion and Conclusion: Our case was 15 months old which is the age of presentation as mentioned internationally. Mesenteric cystic Lymphangiomas are more common among male unlike our case. Ultrasonography and CT abdomen were highly recommended to achieve early diagnosis and treatment of such cases.
\end{abstract}

Keywords: Mesenteric Cystic Lymphangioma; Female; Sudan

\section{Introduction}

Cystic lymphangioma is a benign congenital malformation of the lymphatic vessels. It is due to lack of lymphatic vascular connections; as a result of a developmental defect in the lymphatic system that leads to a sequestration of the lymphatic tissue. It is characterized by an abnormal dilatation and proliferation of lymphatic spaces [1-14]. Lymphangiomas are a group of vascular hamartomas, which result from a developmental failure in the vascular system, including lymphatic and/or arteries and veins. These lymphatic tumors are classified into:

- Simple with capillary lymphatic channels.

- Cavernous with dilated lymphatic and the presence of capsule.

- Macro cystic malformations, clinically termed "cystic hygroma, which is the most common type, and the most affected sites are head and neck.

\footnotetext{
* Corresponding author: Omer Taha Ahmed Elmukashfi

General Surgery Council, Sudan Medical Specialization Board, Khartoum, Sudan
}

Copyright (C) 2021 Author(s) retain the copyright of this article. This article is published under the terms of the Creative Commons Attribution Liscense 4.0. 
The main differential diagnosis is hemangioma, branchial cysts, lipomas and rhabdomyosarcoma [11]. Its first describe by Benevienae in 1507, and there were about 820 cases reported in the literature until 1993[11]. In some literature it is described by Koch, one century ago [1]. Mesenteric cystic lymphangioma (MCL) is a rare condition of unknown etiology, commonly occurred among children, where $60 \%$ appear at birth, and $40 \%$ at one year of age. MCL represents $5-6 \%$ of benign tumors among children. It occurs more in boys than girls (5:2) with a mean age at presentation of 2 years. It has incidence of 1 per 250,000 and affect male more than female. Symptomatic mesenteric cyst accounts for only 1 in 100,000 in acute adult and 1 in 20,000 in acute pediatric admission [1-3, 5, 7, 9-17]. The patho-physiology of MCLs are not clear yet but many theories were put forward like the embryonal developmental theory which could the most accepted one because most of the case diagnosed during childhood. Cystic change can result from any obstruction formed by hemorrhagic or an inflammatory process in the lymphatic vessels $[2,8,11]$. They can be found anywhere in the mesentery along the gastrointestinal tract and can extend from the base of the mesentery into the retro peritoneum. The common sites for lymphangioma are head and neck (75\%), axilla (20\%) in children and it's rare to be found inside the abdomen. The abdominal locations are variables, involving mesentery, mesocolon, retroperitoneum, omentum, splenic loge, liver and pancreas. MCLs are commonly cystic in nature [1, 3-11,14]. MCL has silent clinical course and therefore it's hard to be diagnosed. The clinical features of MCLs range from asymptomatic to acute abdomen. Patients usually present with abdominal pain, abdominal distension, palpable abdominal mass, nausea, vomiting, early satiety, and alteration in bowel habits. These features are related to the cyst dimensions, localizations and relation to the surrounding tissues $[2,5,8,10,14-16,18]$. MCLs can lead to severe complications such as intestinal obstruction, volvulus, ischemia, extrinsic compression, entrapment or peritonitis from ruptured, torsion, and hemorrhage into the cyst or an infected cyst. In relation to abdominal presentations of MCLs, the differential diagnosis includes appendicitis, pancreatitis and even malignancies [2, 4, 9, 14, 15]. Mesenteric cystic lymphangioma may be found coincidentally on physical examination or during imaging. The best diagnostic method for MCLs include: Ultrasonography, computed tomography and magnetic resonance imaging scan and celioscopy. Ultra sound is the best choice for follow up. The diagnosis is confirmed by histopathology $[2-4,13,14,18]$. The gold standard treatment for MCLs is surgical resection including enucleation when feasible, segmental resection of the bowel achieved when the cyst adherent to the bowel through explorative laparotomy or laparoscopy approach. It's first excised by Tillaux (quoted Chung) only in 1802. Surgical excision should be complete for the whole involved part to achieve excellent prognosis; however incomplete excision can lead to recurrence $[2-4,6,10,11,13,18]$.

\section{Case report}

Fifteen months old girls with no significant past medical or surgical history, outcome of normal vaginal delivery at home, attended by midwife, she cried immediately and passed meconium within the first 24 hours. Patient referred to our center for further assessment and investigation regarding diagnosis of abdominal mass. The patient was complained of abdominal distention for 10 days which started gradually, localized and occupying the epigastric region as noticed by her mother. It is associated with mild intermittent fever with no rigor or convulsion. It is not associated with abdominal pain, constipation, vomiting, diarrhea or jaundice. Patient had no history of anorexia or weight loss. Systemic review was unremarkable. On examination: Looks unwell, afebrile, not pale or jaundice. Abdominal examination show: Distended abdomen with full flanks, lobulated mass located within the epigastric region, no area of tenderness and no palpable organomegaly. Investigation: HB: $21.4 \mathrm{~g} / \mathrm{dl}, \mathrm{WBC}: 9.7$, PLT: 468*10, Blood Urea: $19 \mathrm{mg} / \mathrm{dl}, \mathrm{S}$. creatine: 0.3mg /dl, U/G: Clear, S. Na:140 mg/dl, S.K: 4.7meq/dl, Abdomino-pelvic U/S show: very huge abdomino -pelvic cystic mass with multiple thick septa, Ovarian mass? Mesenteric mass? CT abdomen show: huge abdomino-pelvic mass with multiple cysts that having variable sizes measuring up to $6^{*} 7 \mathrm{~cm}$, no other significance. The diagnosis was put as a mesenteric cyst and an elective explorative laparotomy was done. Intra operative findings: Multiple cystic masses arise from the root of the small bowel mesentery matted together with multiple enlarged mesenteric lymph nodes involving segment of distal jejunum with multiple adhesions Figure (1), (2). Procedure: release of adhesion, resection of the masses along with about $57 \mathrm{~cm}$ of the involving jejunum, figure (3), anastomosis was done, and homeostasis was secured. There were no intra operative complications. Specimen was sent for histopathology and the result showed: dilated cystic spaces; lined by flat endothelial cells and multiple reactive lymph nodes, no evidence of malignancy. Features are in line with lymphangioma and associated mesenteric lymphadenitis. Patient discharged on good condition and came to our refer clinic after six weeks and she was quite well. 


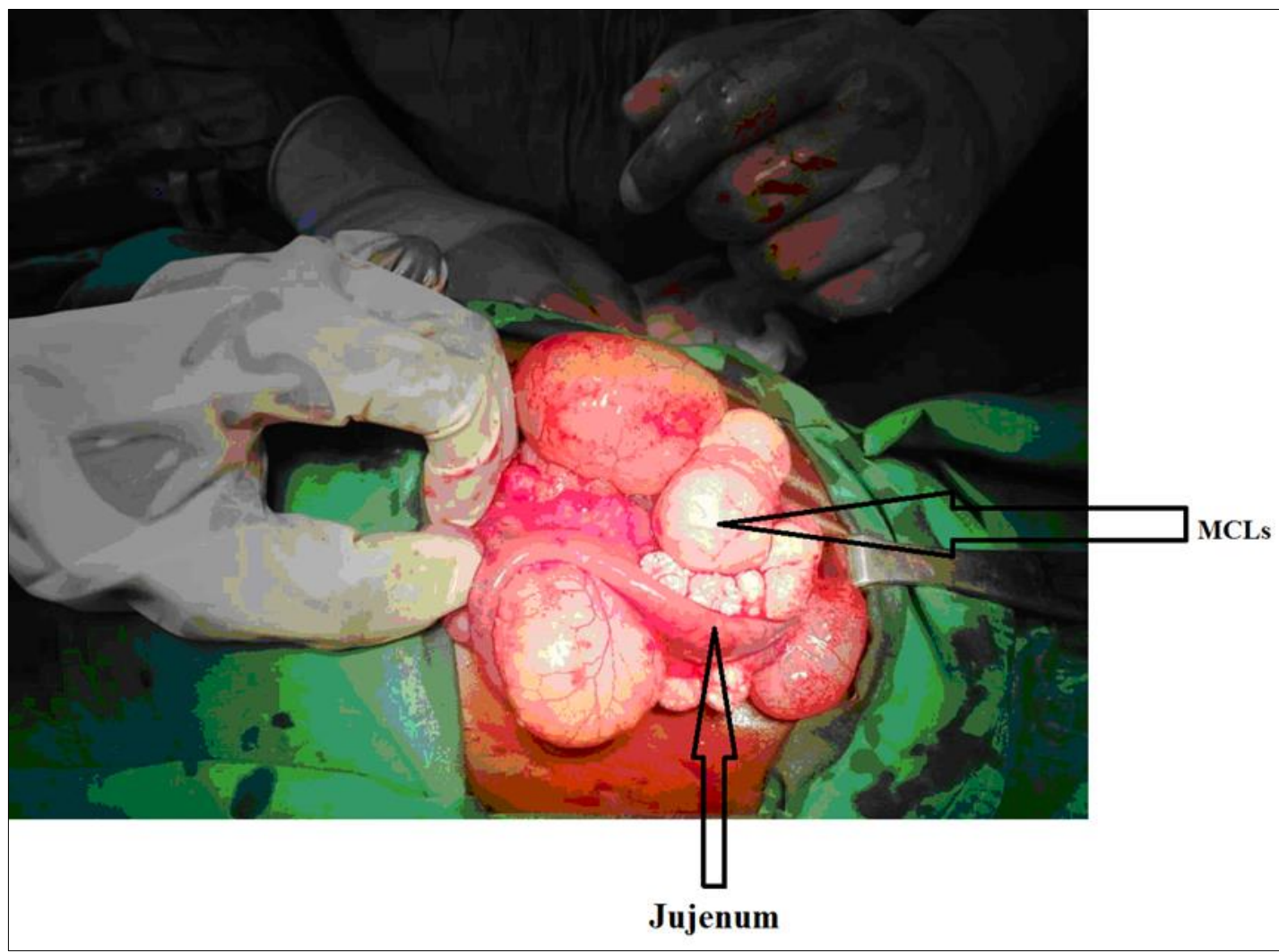

Figure 1 Mesenteric Cystic Lymphangioma arise from the mesentery and adherent to distal jejunum, a rare case report; National Center for Pediatric Surgery, Gezira State, Sudan; 2021

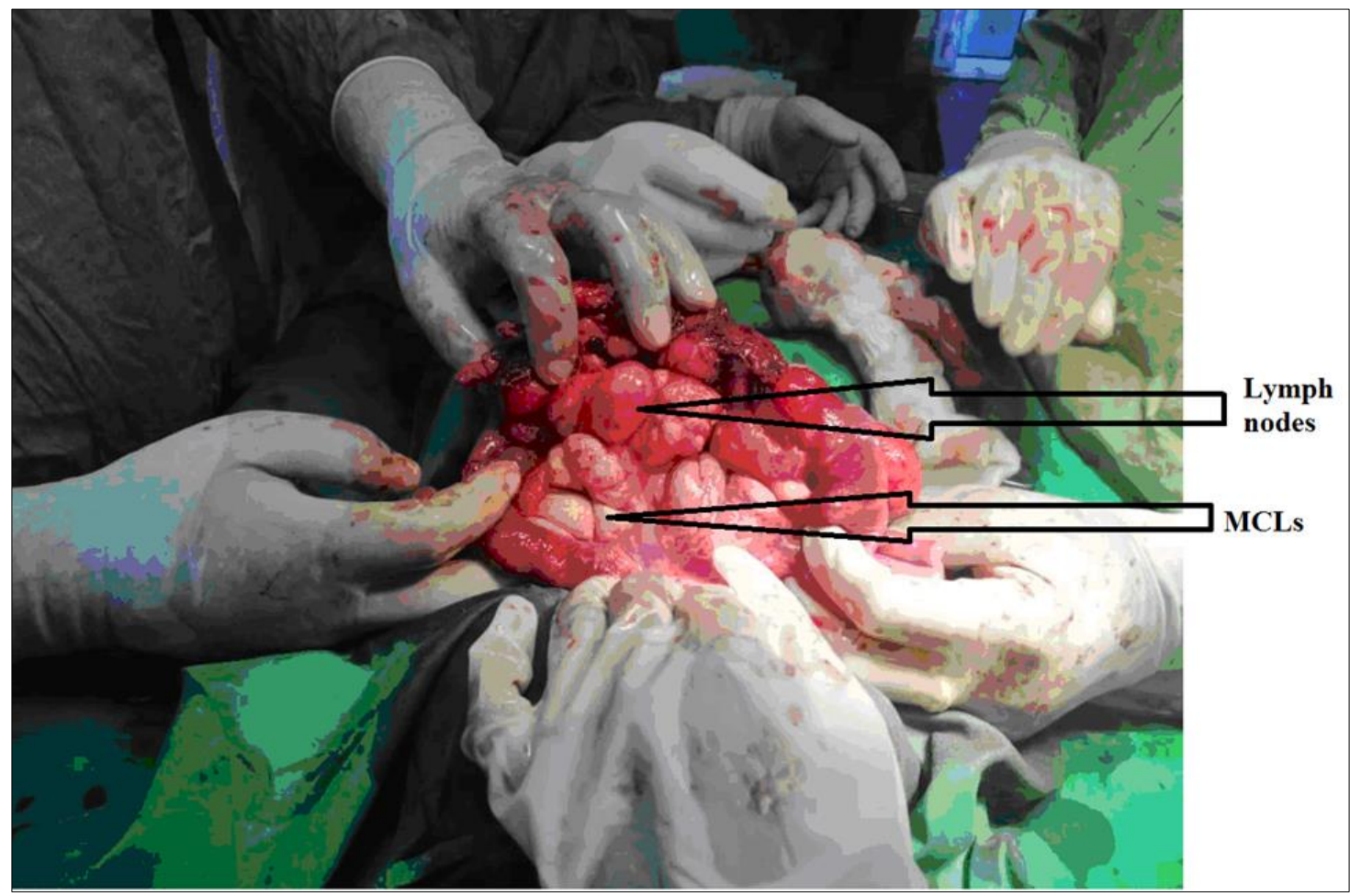

Figure 2 Mesenteric Cystic Lymphangioma matted with multiple mesenteric lymph nodes and adherent together with distal jejunum, a rare case report; National Center for Pediatric Surgery, Gezira State, Sudan; 2021 


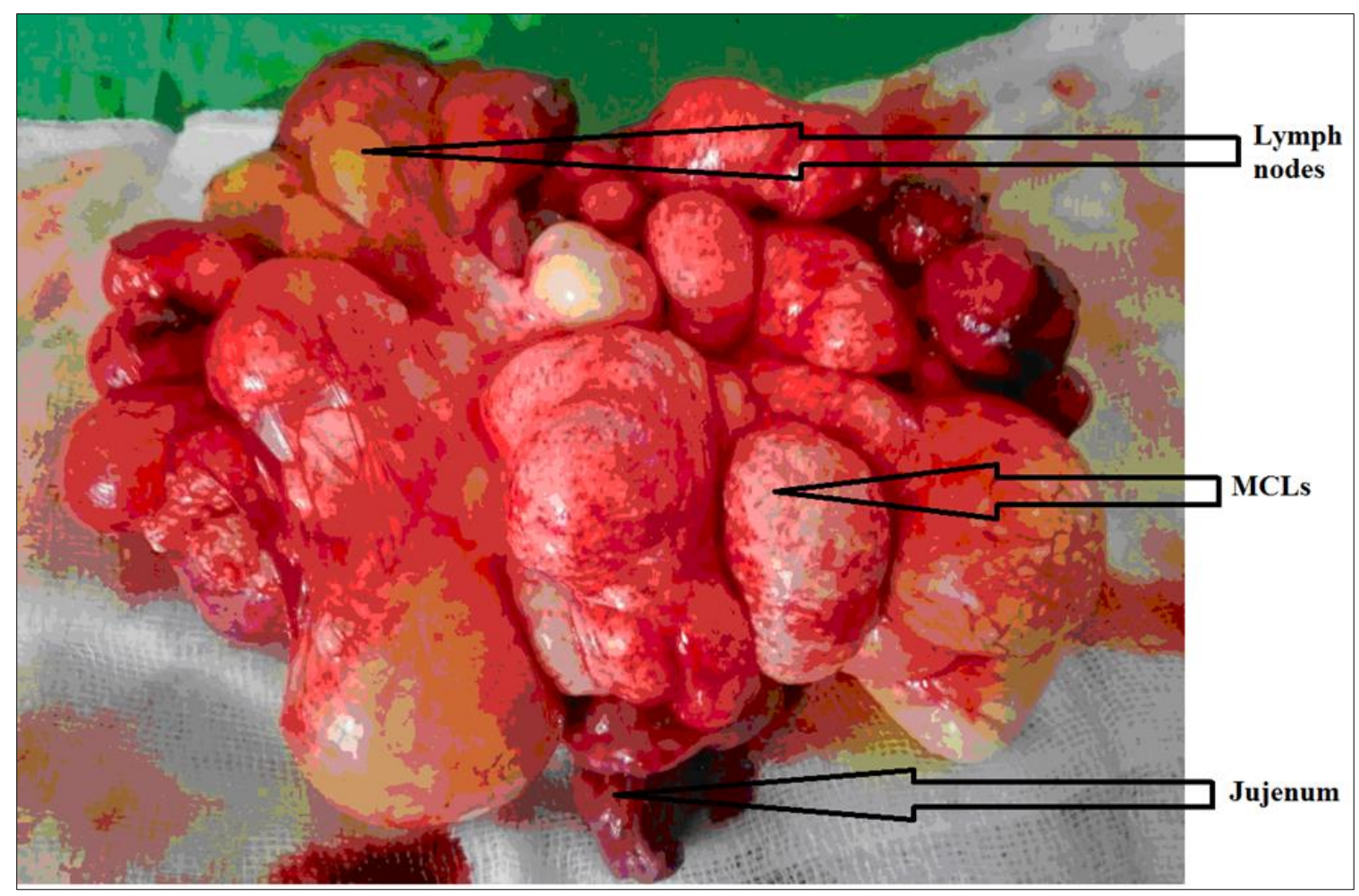

Figure 3 Mesenteric Cystic Lymphangioma with matted mesenteric lymph nodes along with resected part of the jejunum, a rare case report; National Center for Pediatric Surgery, Gezira State, Sudan; 2021

\section{Discussion}

Our case was 15 months old girl and was diagnosed as MCLs which are benign congenital malformation of the lymphatic vessels, that occur due to lack of lymphatic vascular connections as a result of a developmental defect of the lymphatic system during the embryological life resulting in a sequestration of the lymphatic tissue and characterized by an abnormal dilatation and proliferation of lymphatic spaces, [1 - 12]. She was 15-month-old which it's the age of presentation as mentioned internationally [1 - 3, 7, 9 - 17]. MCLs are more common among male unlike our case [5, 10]. Our case had no history of abdominal pain which similar to other studies $[9,11]$. MCLs are very rare to be found inside the abdomen however in our case it's located within the mesentery $[1,3-11,14]$. Our case shows vague presentation which is similar to many case reports $[2,5,10,14-16,18]$. Our case treated by complete surgical excision for the involved part and show good outcome, and this was in line with many other reports $[2-4,6,10,13,18]$. Ultrasonography and CT abdomen were highly recommended to achieve early diagnosis and treatment of such cases.

\section{Conclusion}

The sex of this case was female and her age was less than that of the usual age for presentation of this disease, which is two years. The location site of this tumor was intra-abdomen (within the mesentery). This study highlights the consideration of occurrence of MCLs among female children of less than two years and inside the abdomen.

\section{Compliance with ethical standards}

\section{Disclosure of conflict of interest}

There is no conflict of interest

\section{Statement of informed consent}

Informed consent was obtained from the parents of the patient. 


\section{References}

[1] Boume MA, et al, Unilocular Cystic Lymphangioma of the Small Omentum in a Girl of 4 Years, Clinics in Surgery, 2017; 2(1487): 1-4.

[2] Abdulraheem AK, Al Sharie AH, Al Shalakhti MH, Alayoub SY, Al-Domaidat HM, El-Qawasmeh AE, Mesenteric cystic lymphangioma: A case report, International Journal of Surgery Case Reports. 2021; 80: 105659.

[3] Gunadi, et al, Pediatric patients with mesenteric cystic lymphangioma: A case series, International Journal of Surgery Case Reports. 2019; 64: 89-93.

[4] Mahmoudi A, Rami M, Khattala K, El Madi A, Bouabdallah Y, Huge omental lymphangioma with haemorrhage in children: case report, Pan African Medical Journal. 2020; 35(20): 1-6.

[5] Gallart RM, Boga AS, Tellado MG, Argibay IS. Giant mesenteric cystic lymphangioma in an infant presenting with acute bowel obstruction, Can J Surg. June 2009; 52(3): E42-E43.

[6] Minocha P K, Roop L, Persad R, Cases of Atypical Lymphangiomas in Children, Case Reports in Pediatrics. 2014; 6.

[7] Rathod j, Patel S, Upadhyay H M, Cystic lymphangioma of the jejunal mesentery in a young adult: a rare case report, Int Surg J. Nov 2020; 7(11): 3831-3834.

[8] Kiran RS, Sarmukh S, Azmi H, Mesenteric Cystic Lymphangioma "Intra-abdominal Catastrophe, Asian Journal of Advanced Research and Reports. 2020; 14(3): 1-5.

[9] Vega IF, Cystic Lymphangioma: an Uncommon Cause of Pediatric Abdominal Pain, International Journal of Pathology and Clinical Research. 2017; 3:053: 1-4.

[10] Katı O, Güngör S, Kandur Y, Mesenteric cystic lymphangioma: Case report, Journal of Pediatric Surgery Case Reports. 2018; 35: 26-28.

[11] Bendjaballah A, et al, Cystic Lymphangioma of the Mesentery: Difficulties of Diagnosis and Surgical Treatment Outcome, International Journal of Surgical Research. 2018; 7(1): 1-6.

[12] Rattan KN, Nair VJ, Pathak M, Kumar S, Pediatric chylolymphatic mesenteric cyst - a separate entity from cystic lymphangioma: a case series, Journal of Medical Case Reports. 2009; 3(111): 1-4.

[13] Khattala K, Rami M, Elmadi A, Mahmoudi A, Bouabdallah Y, Giant cystic lymphangioma of the small bowel mesentery: case report, Pan African Medical Journal. 2011; 9(46): 1-5.

[14] Kusuma FSP, Chylous mesenteric cyst in children -A case report in a 4-year-old boy, Med J Malaysia. 1 May 2020; 75: 48-50.

[15] Chen J, Du L, Wang R D, Experience in the diagnosis and treatment of mesenteric lymphangioma in adults: a case report and review of literature, World J Gastrointest Oncol. December 2018; 10(12): 522-527.

[16] Rathod J, Yadav H, Arora D, Cystic lymphangioma of small bowel mesentery: a rare case report, International Journal of Research in Medical Sciences. July 2015; 3(7): 1782-1784.

[17] Maranna H, et al, Cystic Lymphangioma of the Greater Omentum: A Case of Partial Spontaneous Regression and Review of the Literature, Case Reports in Surgery. 2020; 5.

[18] Er A, Kaymakcıoğlu N, Çerci C, Giant Abdominal Mesenteric Cyst, European Journal of General Medicine. 2009; 6(3): 189-193. 